\title{
Archéopages
}

Archéopages Archéologie et société

42 | 04-07/2015

Construire en terre crue

\section{Constructions médiévales en torchis}

Étude de fragments issus de la démolition d'un bourg de la plaine du Doubs

Medieval wattle and daub buildings. A study of the remains from the demolition of a settlement in the plain of the Doubs

Construcciones medievales de entramado vegetal con mortero de tierra. Estudio de fragmentos obtenidos tras la demolición de un burgo de la planicie del Doubs

\section{Christophe Meloche et Dominique Sordoillet}

\section{OpenEdition}

Journals

Édition électronique

URL : https://journals.openedition.org/archeopages/1283

DOI : $10.4000 /$ archeopages. 1283

ISSN : 2269-9872

\section{Éditeur}

INRAP - Institut national de recherches archéologiques préventives

Édition imprimée

Date de publication : 1 mars 2016

Pagination : 100-105

ISSN : 1622-8545

Référence électronique

Christophe Meloche et Dominique Sordoillet, «Constructions médiévales en torchis », Archéopages [En ligne], 42 | 04-07/2015, mis en ligne le 01 juillet 2017, consulté le 03 juin 2021. URL : http:// journals.openedition.org/archeopages/1283; DOI : https://doi.org/10.4000/archeopages.1283 


\section{Constructions médiévales en torchis Étude de fragments issus de la démolition d'un bourg de la plaine du Doubs}

\section{Christophe Méloche Inrap}

avec la collaboration de Dominique Sordoillet Irrap, UMR 6249 «Chrono-environnement,
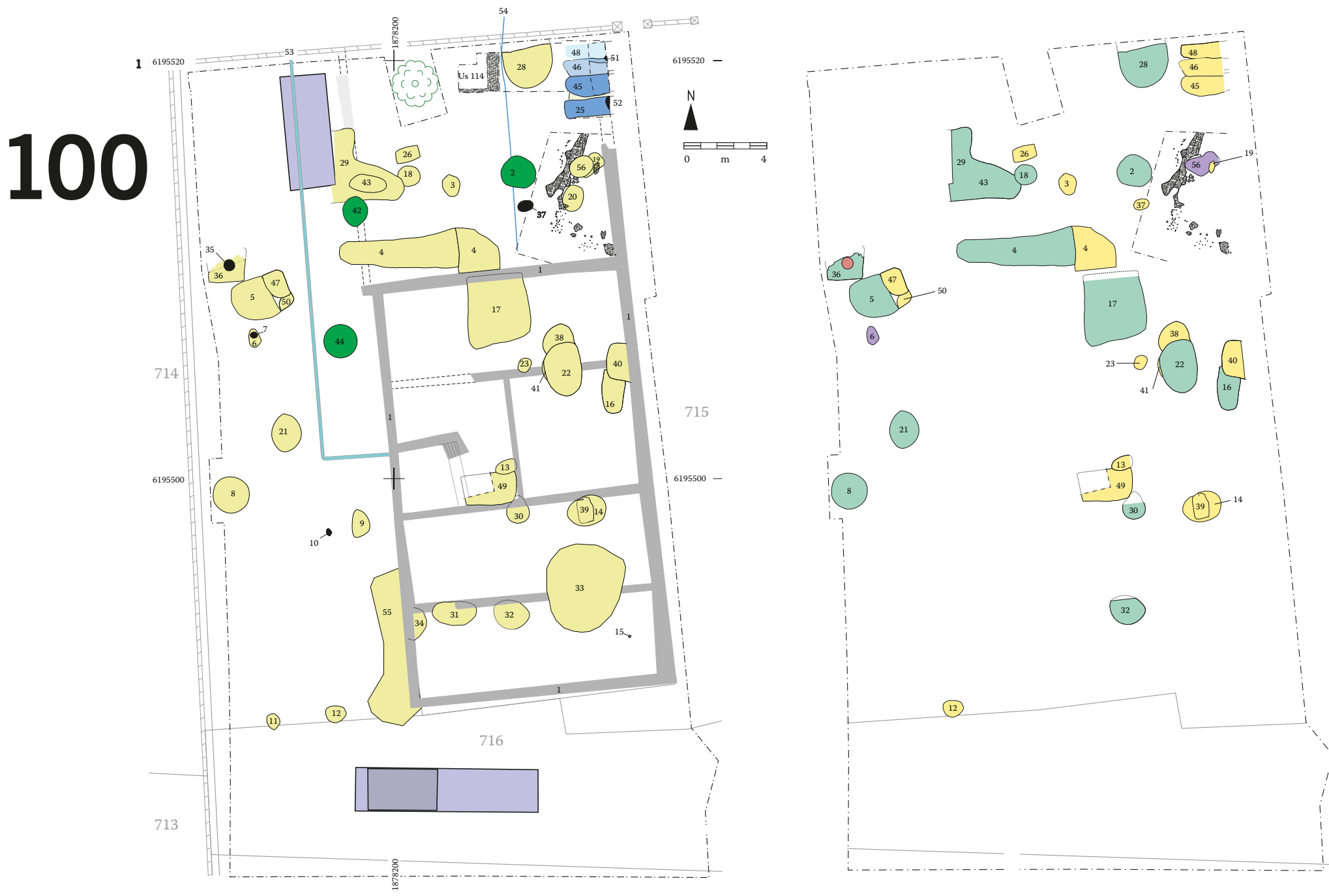

1. Relevé de l'ensemble des structures et répartition des structures ayant livré des fragments de torchis et de pisé entre le XIII'-xIV siècle et la période moderne. La fouille a mis en évidence une zone

comprenant sans doute, au xIII'-xive siècle, deux jardins distincts occupant la surface de parcelles étroites. Les habitats associés seraient situés plus au nord, en partie sous l'actuelle chaussée. La capacité de stockage cumulé des nombreux silos et fosses attribués à cette occupation atteint $11 \mathrm{~m}^{3}$.
Sondages profonds

Murs bâtiment XIX ${ }^{\mathrm{e}} \mathrm{s}$

- Structures en creux

Adduction et évacuation des eaux

- - Limite de décapage
Structure du $\mathrm{XIV}^{\mathrm{e}} \mathrm{s}$. dont le remplissage a livré du torchis ou du pisé

Structure du XIV ${ }^{\mathrm{e}}$ s. dont le remplissage n'a pas livré du torchis ou du pisé

Structure du XIII ${ }^{\mathrm{e}}$-XVIII ${ }^{\mathrm{e}}$ s. dont le remplissage a livré du torchis ou du pisé

Structure non datée dont le remplissage a livré du torchis ou du pisé 
Le village de Petit-Noir (Jura) se situe dans la basse vallée du Doubs, à 185 m d'altitude et à environ $1 \mathrm{~km}$ du cours principal de la rivière, qui forme ici un coude après avoir butté contre les reliefs bressans. Cette commune localisée dans l'extrémité nord-ouest du département, limitrophe à la Saône-et-Loire, se trouvait sur la frontière des duché et comté de Bourgogne.

$\mathrm{Au} \mathrm{XIII}{ }^{\mathrm{e}}$ siècle, le bourg était protégé par une enceinte en U s'appuyant à l'est contre le méandre alors actif du Doubs. Son tracé a laissé une empreinte que tous les documents cadastraux depuis 1758 ont fidèlement enregistrée. L'espace enclos est structuré par un réseau viaire orthonormé découpant des îlots réguliers. Les auteurs du XIX ${ }^{\mathrm{e}}$ siècle signalaient la présence d'au moins deux portes fortifiées dont nous n'avons pu encore retrouver la mention dans les documents d'archives consultés. Le bourg enclos était partagé entre plusieurs seigneurs. La part de l'abbaye de Château-Chalon était dite la " petite seigneurie », l'autre qui était tenue par les descendants de Robert de Bourgogne puis par les familles de Rye et de Broissia se nommait la « grande seigneurie». Seuls les sujets de cette dernière seigneurie bénéficiaient d'une charte de franchise qui leur fut accordée en 1272.

C'est à l'est de l'emplacement présumé d'une des portes qu'un premier diagnostic, en $2011^{\mathbf{1}}$, a mis en évidence une occupation médiévale le long de l'actuelle rue de la Mairie (Billoin, 2012). Un diagnostic complémentaire en 2013 a permis de sonder une vaste zone vide de structures s'étendant sur plus de $100 \mathrm{~m}$ au sud de la parcelle sondée en 2012 (Méloche, 2013). La fouille ${ }^{2}$ menée sur une superficie de $74.1 \mathrm{~m}^{2}$ a concerné plus de 50 structures dont plus des neuf dixièmes ont été datées par un très abondant mobilier céramique du XIII ${ }^{\mathrm{e}}$ et XIV ${ }^{\mathrm{e}}$ siècle (Méloche, 2015) [ill. 1]. Il n'y a aucune structure antérieure au Moyen Âge central et l'époque moderne n'a laissé que des traces fugitives. Deux grandes fosses ont servi d'approvisionnement en matériaux de construction pour l'édification de murs en torchis, dont de nombreux vestiges ont été retrouvés lors de la fouille :2,377 kg ont été prélevés pour étude et, dans ce lot, à une exception près, tout provient des structures datées des XIII ${ }^{\mathrm{e}}$-XIV ${ }^{\mathrm{e}}$ siècles.

\section{Le contexte de la fouille}

Cette zone de jardin comprenait deux puits. L'examen du plan terrier de 1758 permet de replacer avec certitude la partie de la parcelle fouillée dans le ressort de la "grande seigneurie». Pour la période moderne, seuls quelques lambeaux de sol et de rares fosses d'équarrissage sont connus. Entre 1758 et 1824, une maison fut bâtie perpendiculairement à la rue, à l'emplacement des vestiges médiévaux qu'elle ne perturbe que faiblement. Le long des rues, on trouvait l'habitat et, en profondeur, une zone de jardins auxquels succédaient des champs cultivés. La disproportion des zones cultivées par rapport aux superficies bâties ne doit pas faire illusion : il s'agit bien d'un bourg doté de son enceinte, de son marché, de sa charte de franchise et des institutions communales qui en ont découlé, et, en l'absence de château, de bâtiments représentant le pouvoir seigneurial comme la prison et la salle de justice.

Ce village a été bâti au cour d'un ancien méandre où coule actuellement le ruisseau dit de l'Échappée. Le sous-sol est naturellement constitué d'alluvions du Quaternaire (Fza), dont les faciès les plus récents, ceux qui nous concernent ici, sont décrits comme des sédiments fins, argileux, marneux ou sableux, de couleur brune à beige, selon que l'argile ou le calcaire prédomine (Fleury et al., 1984). Un sondage du Bureau de recherches géologiques et minières indique que ces alluvions atteignent au moins 3,7 m d'épaisseur à cet endroit.

Les sondages effectués à l'occasion du diagnostic archéologique confirment la nature alluviale du terrain et révèlent plusieurs épisodes de remblaiement liés aux travaux d'aménagement médiévaux ou plus récents (Billoin, 2012, fig. 5). Les argiles de crue, plus ou moins chargées de limons et de sables, apparaissent à une profondeur variant entre o,55 et o,75 $\mathrm{m}$ sous la surface actuelle du sol. Assez régulières d'est en ouest, elles montrent une légère pente vers le sud, vers la sortie du méandre. Ces alluvions fines sont recouvertes d'abord par des déblais sablo-graveleux riches en fragments de tuile et de poterie médiévale, puis beaucoup plus récemment par des remblais sablograveleux. Le profil se termine par l'horizon de terre végétale.

En l'absence de dépôt de crue ou de rigole d'érosion clairement lisibles, on peut penser que le secteur sondé est resté relativement à l'abri des inondations au cours des XIII ${ }^{\mathrm{e}}-\mathrm{XIV}^{\mathrm{e}}$ siècles. Trente structures appartiennent à cet état, auxquelles s'ajoute un niveau de remblai. Elles se répartissent en silos, fosses diverses, puits et rares trous de poteau. Si aucun plan de bâti n'a pu être mis en évidence, ce sont les restes de mobilier contenus dans les remplissages des fosses et silos qui indiquent non seulement la proximité d'un bâtiment, mais encore plus d'un habitat comme le prouve la présence d'un grand nombre de couvrefeu rappelant l'existence de foyers domestiques.

\section{Les constructions en torchis}

Rares sont les structures qui n'ont pas livré de fragments de torchis rubéfiés ou couverts de suie. L'exception la plus notable est la zone des « fossés » nord-est dont les remplissages n'en ont livré aucun. Il en est de même pour le silo 47 situé dans la partie nord-ouest de l'emprise [ill. 2]. On trouve ces éléments de parois et de cloisons dans des structures très riches en rejets cendreux, c'est-à-dire dans le cas où l'un des comblements est une couche saturée de charbons de bois (cas des structures ${ }^{3}$ 2, 17 et 22) [ill. 3]. Les éléments de paroi récoltés sont exclusivement des restes de torchis. Par ailleurs, l'importante direction de David Billoi 2. Opération menée en avril 2014 sous la direction de Christophe Méloche. 3. Le « fossé » 45 faisant encore exception. 




$\rightleftharpoons \underset{\mathrm{m}}{\rightleftharpoons}$

St. 5

Limon argileux brun avec des inclusions de plaques de torchis rubéfiés très fragmentés

Limon noir cendreux avec des inclusions d'argile rubéfiée et noircie

Limon ocre avec des fragments de torchis rubéfiés

Limon cendreux gris

Limon de nature hétérogène de teinte grisâtre

Niveau de terre rubéfiée rouge vif homogène

Niveau de terre rubéfiée violacée avec des inclusions de fragments de torchis Poche de fragments de torchis rubéfiés

Limon argileux grisâtre cendreux compact

Niveau proche du T.N : limon argileux jaunâtre marbré de gris

Vestige de paroi ? : plaquage d'argile rubéfiée très compacte

St. 47

Limon de nature hétérogène brun gris avec des inclusions de charbons de bois de taille centimétrique et de nodule d'argile rubéfiée Limon argileux brun jaunâtre avec des inclusions de poches et lentilles charbonneuses et des inclusions de torchis rubéfiés

$\square$ Limon argileux grisâtre (dans lequel reposait la carcasse)

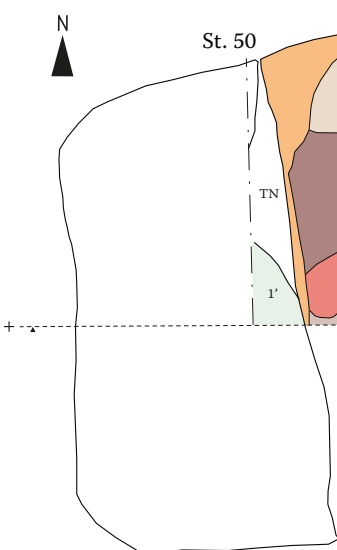

St. 47

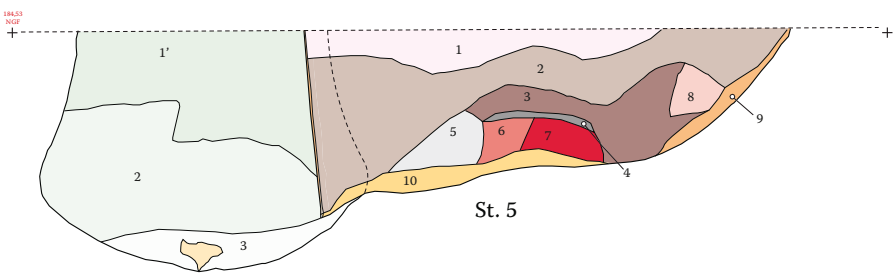

St. 47

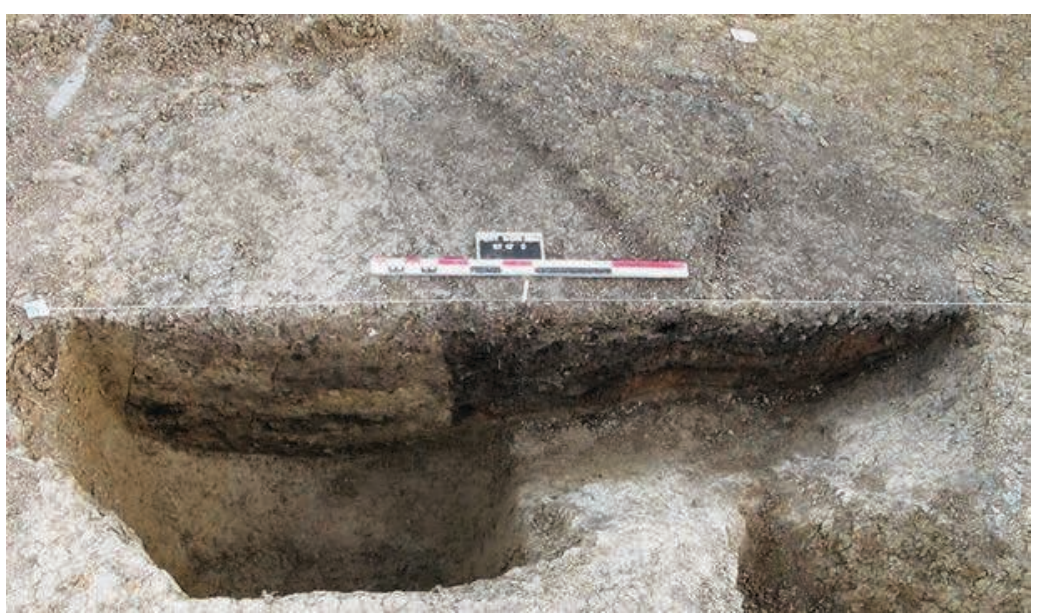

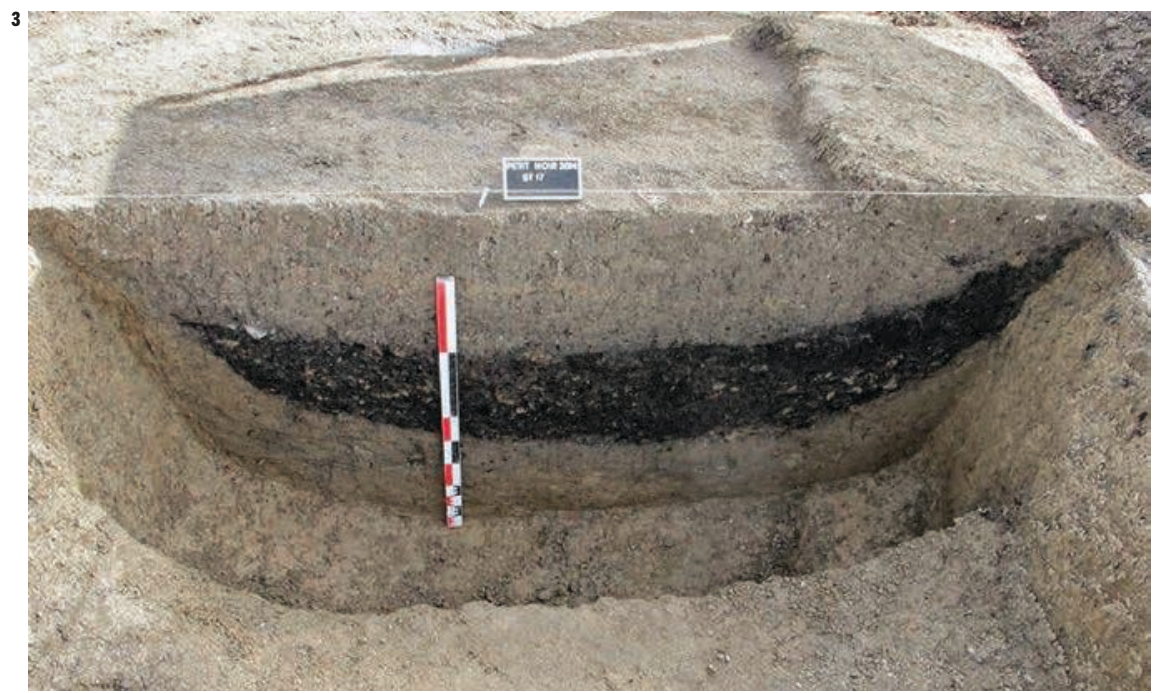

3. La couche cendreuse

de cette fosse

d'extraction (st.17)

comprenait de nombreux

vestiges de constructions

en torchis. 

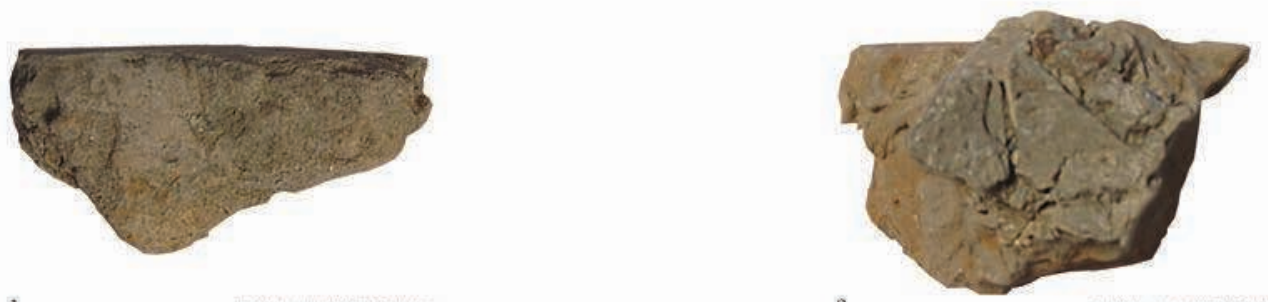

gravillons.


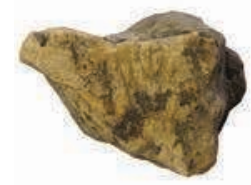

4



5



St 2 Us 1, lot 3857 MC 0016
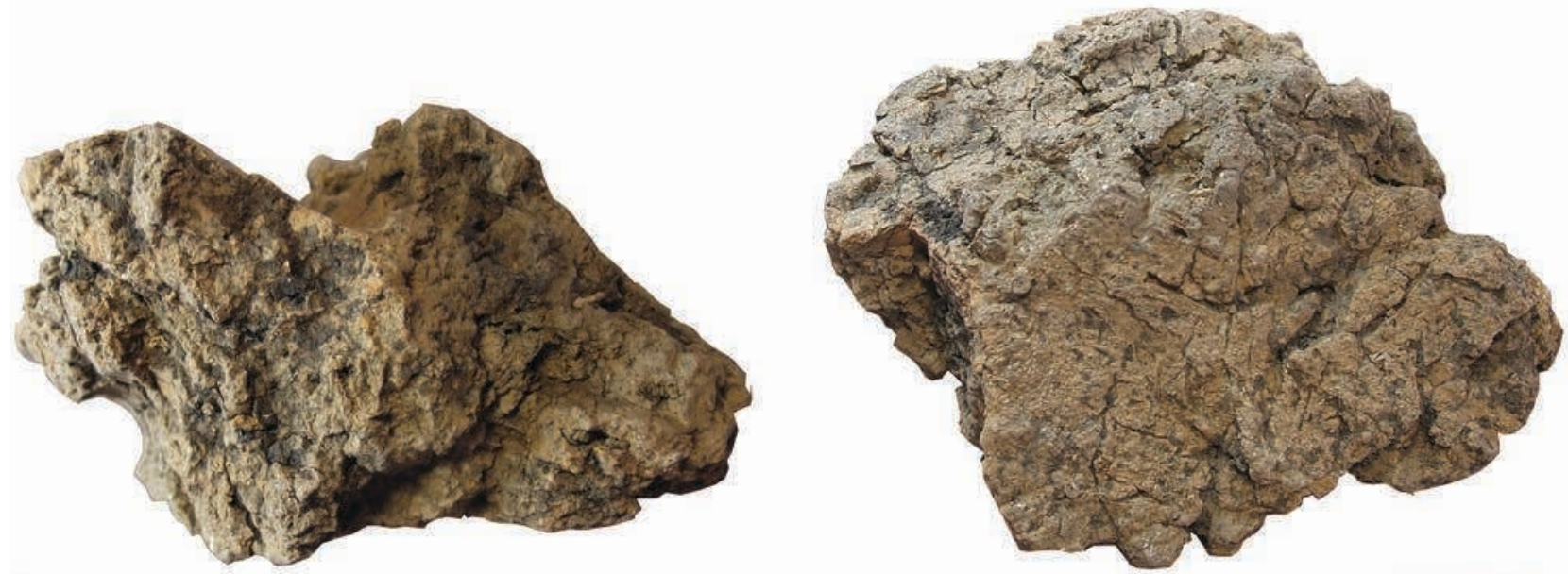
teneur de ces matériaux en sable d'origine calcaire montre qu'ils ont été prélevés dans le substrat local. Ainsi, au centre de l'emprise, la fosse 17, qui possède un volume estimé à $12 \mathrm{~m}^{3}$, a pu être interprétée comme une fosse d'extraction d'un limon argileux très riche en sable.

Les torchis étudiés peuvent être appliqués par mottes [ill. 4]. Recouvrant l'armature des panneaux constitués par le clayonnage, le torchis peut être simplement plaqué. Sa surface extérieure correspondant au nu du mur ou de la paroi est alors à peine égalisée. Le même effet est obtenu avec un sédiment plus riche en sable pour les torchis pauvres en inclusions de matières végétales. Parfois les surfaces sont talochées et soigneusement lissées. On peut distinguer, en coupe, l'existence d'une première couche constituée par des argiles siliceuses serrées avec de rares inclusions de graviers calcaires millimétriques. Lorsque deux faces opposées du même fragment sont lissées (7 cas sur 87 fragments étudiés), l'épaisseur moyenne de ce lot est de 4,1 cm. L'épaisseur moyenne pour tout le lot collecté est de $3,3 \mathrm{~cm}$.

La paroi pouvait recevoir un véritable enduit. C'est le cas pour trois fragments. Cet enduit peut être un mortier de chaux d'une épaisseur de $2,3 \mathrm{~mm}$ qui recouvre une couche d'argile agglomérée avec des inclusions de fragments de terre cuite architecturale et de végétaux. Pour l'un des fragments retrouvés dans le puits 2, le mortier de chaux ayant chauffé a pris une teinte rosée. Dans ce dernier cas, la couche de mortier avait une épaisseur de $8 \mathrm{~mm}$. On peut supposer que ce revêtement appartenait à un mur à pan de bois en position de gouttereau ou de pignon appartenant à une habitation. Si un tel enduit pourrait trahir un certain souci esthétique, ou du moins le statut social du propriétaire, il permettait surtout d'assurer une longévité au mur ainsi protégé.

Les empreintes des baguettes du clayonnage ont pu être relevées dans 15 cas. Leur diamètre est compris entre o,6 et $2 \mathrm{~cm}$. Dans un cas, la disposition des surfaces planes permet d'identifier un fragment provenant d'une construction en pan de bois mettant en œuvre des décharges.

Si les rejets dans les fosses et silos attestent bien d'une construction médiévale qui semble, dans cette partie du bourg, être, en l'absence de tout moellon, exclusivement en pan de bois hourdé de torchis, il est à noter que les éléments de toiture sont pratiquement totalement absents. Une seule structure (St 32) a livré pour le XIII ${ }^{\mathrm{e}}$ siècle un fragment de tuile plate droite, dont le crochet est brisé. Les trois autres tuiles plates retrouvées appartiennent à la période moderne, notamment une tuile glaçurée provenant d'un remblai. Un fragment de tuile canal, sans doute à crochet, provient du décapage et ne peut être rattachée à aucune structure. En l'absence également de clou de tavaillon, dont la forme pour cette période est parfaitement caractéristique, il convient de restituer une toiture végétale pour ces bâtiments.

À la fin du Moyen Âge central, les maisons en pierre sont rares en milieu rural, si l'on excepte les granges cisterciennes dont l'architecture liée au monde monastique se rapproche bien plus des modèles urbains. En Franche-Comté, à la charnière entre le Moyen Âge central et le bas Moyen Âge, les murs à pan de bois hourdés de torchis sont la norme pour l'habitat populaire tant en milieu urbain que dans les faubourgs : citons par exemple les maisons du quartier Velotte à Montbéliard (Tchirakadzé, Fuhrer, 1998), les maisons vigneronnes de la rue Serpente à Vesoul (Munier, 2009), de la rue du Vignier à Besançon (Goy et al., 1990), la maison du faubourg Glanot à Pesmes (Bonvalot, 1987). Plus récemment, à Burgille dans le Doubs, un diagnostic réalisé au cœur du village a révélé l'existence d'un petit bâtiment à pan de bois détruit par un incendie au cours du $\mathrm{Xv}^{\mathrm{e}}$ siècle (Nowicki, 2015).

Sur le premier plateau du massif jurassien, on manque d'exemple archéologique. Il y aurait tout de lieu de penser que le matériau immédiatement disponible est fourni par le substrat calcaire affleurant de toute part. Ainsi, les bâtiments de la grange de Crans ${ }^{\mathbf{4}}$, détruits vers 1370 , étaient élevés en moellons liés au mortier de chaux (Méloche, 1994). Cependant, la documentation écrite de la période suivante atteste que, là aussi, la construction en pan de bois hourdée de torchis restait également fréquente : les terriers et aveux de dénombrements rédigés $\mathrm{au} \mathrm{XV}^{\mathrm{e}}$ et $\mathrm{XVI}^{\mathrm{e}}$ siècle dans la région d'Orgelet (Jura), dite la « petite montagne », mentionnent un très grand nombre de maisons « de bois » et « de paille » (Méloche, 2003).

« L'architecture de torchis » semble une évidence pour le bourg de Petit-Noir situé dans la plaine alluviale du Doubs, qui se trouve éloignée d'une bonne trentaine de kilomètres des premières carrières des massifs du Jura ou de la Serre. On peut penser que ce mode de construction perdure jusqu'au début du XIX ${ }^{\mathrm{e}}$ siècle : en 1816, les inondations provoquèrent la destruction de 21 maisons sans doute construites ainsi, comme en témoignent des structures aujourd'hui en élévation dans la même commune [ill. 5]. 


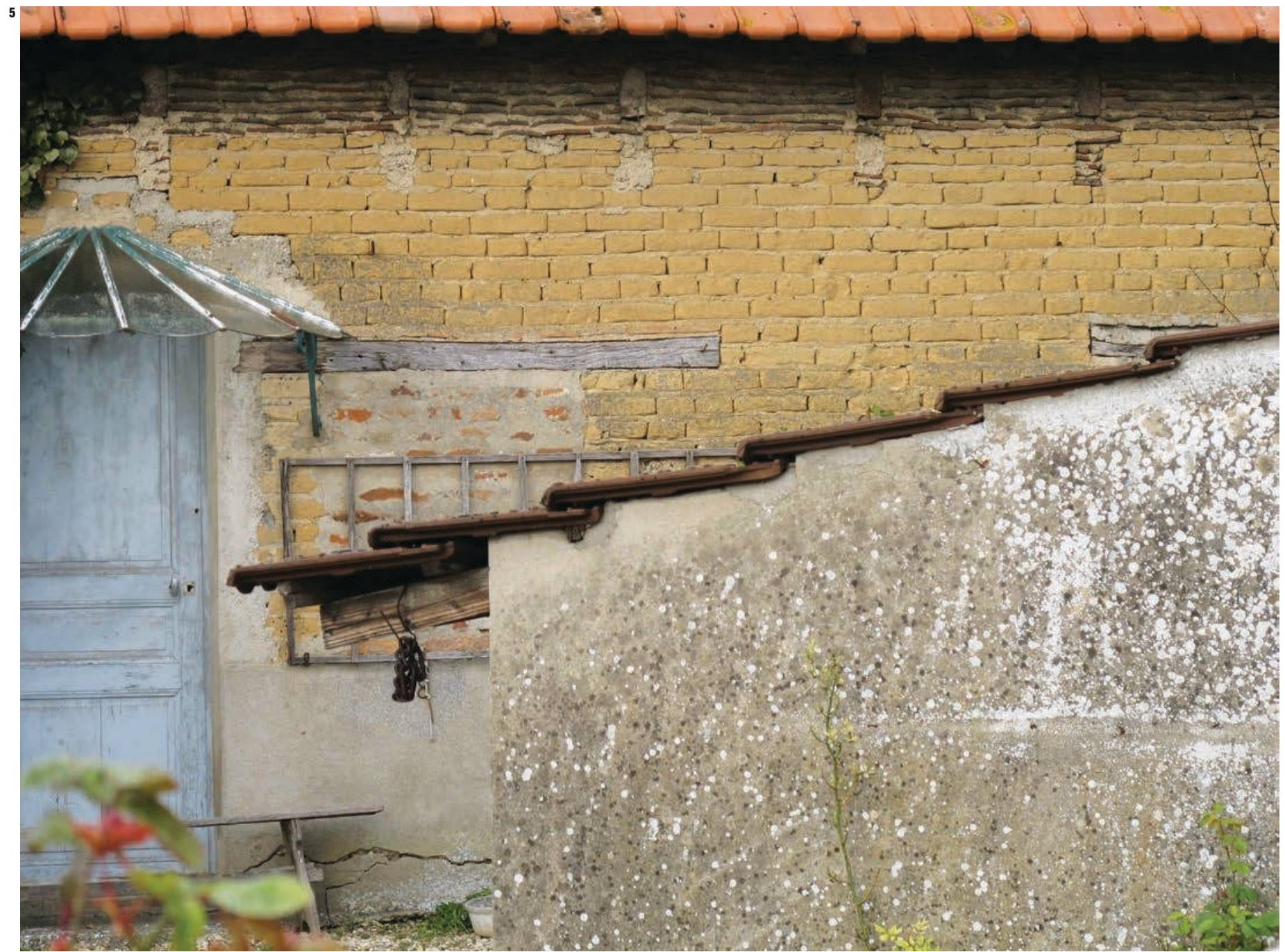

5. Si la maison édifiée au xixe siècle sur l'emprise de la parcelle fouillée possédait des murs en moellons, il subsiste encore sur la commune des traces de cette architecture de terre sous la forme de murs d'adobe.

\section{Références bibliographiques}

Billoin D., 2012, Petit-Noir (Jura), 5 rue de la Mairie. Une occupation médiévale du XIII ${ }^{e}-X V I^{e}$ siècle au centre de lancien bourg castral, rapport d'opération, InrapSRA Franche-Comté, 44 p.

BonvALOT N., 1987, « Habitat rural médiéval hors les murs à Pesmes », Bulletin de la Société d'agriculture, lettres, arts et sciences de la Haute-Saône, p. 49-51.

Fleury R., Clozier L. et al., 1984, Notice explicative de la feuille Pierre-de-Bresse à 1/50ooo, Orléans, éditions du Bureau de recherches géologiques et minières, $40 \mathrm{p}$.

Goy C., Guilhot J.-O., Munier C., Pinette M., 1990, Se nourrir à Besançon au Moyen Âge : à la table d'un vigneron de Battant, Besançon, Musée des Beauxarts, $83 \mathrm{p}$.

MéLOCHE C., 1994, Une grange féodale du XIV s. à Crans (Jura), Mémoire de maittrise en Sciences et Technologie (sous la direction de J.-M. Poisson), Lyon, université de Lyon II, $102 \mathrm{p}$.

MÉLOCHE C., 2003, Les granges laïques des bailliages d'Aval et de Dole au travers de la documentation moderne $X V^{e}-X V I^{e} S$., programme 20, rapport d'activité, SRA Franche-Comté, 275 p.

MéLOCHe C., 2013, Petit-Noir (Jura), 5 rue de la Mairie évaluation du potentiel archéologique de la parcelle $C_{717}$, rapport d'opération, Inrap-SRA FrancheComté, 48 p.

MÉLOCHE C., 2015, Franche-Comté, Jura, Petit-Noir Au Village. Les abords d'un quartier médiéval (XIII'-XIV siècles), rapport d'opération, Inrap-SRA FrancheComté, $226 \mathrm{p}$.

Munier C., 2009, Franche-Comté, Haute-Saône (7o), Vesoul, 4 rue Serpente, rapport d'opération, InrapSRA Franche-Comté, 26o p.

NowICKI P. (DIR.), 2015, Burgille, 21 Grande Rue. Un bâtiment du XV siècle à proximité du château $d u X V I I I^{e}$ siècle, rapport d'opération, Inrap-SRA Franche-Comté, 44. p.

TCHIRAKADZÉ C., FURHER É., 1998, En quête d'une mémoire. 10 ans d'archéologie urbaine à Montbéliard catalogue d'exposition, Châtenois-les-Forges, 128 p. 